\title{
Data Mining and Fusion of Unobtrusive Sensing Solutions for Indoor Activity Recognition
}

DOI:

10.1109/EMBC44109.2020.9175896

\section{Document Version}

Accepted author manuscript

Link to publication record in Manchester Research Explorer

\section{Citation for published version (APA):}

Ekerete, I. F., Garcia-constantino, M., Diaz, Y., Giggins, O. M., Mustafa, M. A., Konios, A., Pouliet, P., Nugent, C. D., \& Mclaughlin, J. (2020). Data Mining and Fusion of Unobtrusive Sensing Solutions for Indoor Activity Recognition. In 2020 42nd Annual International Conference of the IEEE Engineering in Medicine \& Biology Society (EMBC) (pp. 5357-5361) https://doi.org/10.1109/EMBC44109.2020.9175896

\section{Published in:}

2020 42nd Annual International Conference of the IEEE Engineering in Medicine \& Biology Society (EMBC)

\section{Citing this paper}

Please note that where the full-text provided on Manchester Research Explorer is the Author Accepted Manuscript or Proof version this may differ from the final Published version. If citing, it is advised that you check and use the publisher's definitive version.

\section{General rights}

Copyright and moral rights for the publications made accessible in the Research Explorer are retained by the authors and/or other copyright owners and it is a condition of accessing publications that users recognise and abide by the legal requirements associated with these rights.

\section{Takedown policy}

If you believe that this document breaches copyright please refer to the University of Manchester's Takedown Procedures [http://man.ac.uk/04Y6Bo] or contact uml.scholarlycommunications@manchester.ac.uk providing relevant details, so we can investigate your claim.

\section{OPEN ACCESS}




\title{
Data Mining and Fusion of Unobtrusive Sensing Solutions for Indoor Activity Recognition
}

\author{
Idongesit F. Ekerete ${ }^{1}$, M. Garcia-Constantino ${ }^{1}$, Yohanca Diaz ${ }^{2}$, Oonagh M. Giggins ${ }^{2}$, M. A. Mustafa ${ }^{3}$, \\ Alexandros Konios ${ }^{4}$, Pierre Pouliet ${ }^{5}$, Chris D. Nugent ${ }^{1}$, Member, IEEE, Jim McLaughlin ${ }^{6}$, Member, \\ IEEE.
}

\begin{abstract}
This paper proposes the fusion of data from unobtrusive sensing solutions for the recognition and classification of activities in home environments. The ability to recognize and classify activities can help in the objective monitoring of health and wellness trends in ageing adults. While the use of video and stereo cameras for monitoring activities provides an adequate insight, the privacy of users is not fully protected (i.e., users can easily be recognized from the images). Another concern is that widely used wearable sensors, such as accelerometers, have some disadvantages, such as limited battery life, adoption issues and wearability. This study investigates the use of low-cost thermal sensing solutions capable of generating distinct thermal blobs with timestamps to recognize the activities of study participants. More than 11,000 thermal blobs were recorded from 10 healthy participants with two thermal sensors placed in a laboratory kitchen: (i) one mounted on the ceiling, and (ii) the other positioned on a mini tripod stand in the corner of the room. Furthermore, data from the ceiling thermal sensor were fused with data gleaned from the lateral thermal sensor. Contact sensors were used at each stage as the gold standard for timestamp approximation during data acquisition, which allowed the attainment of: (i) the time at which each activity took place, (ii) the type of activity performed, and (iii) the location of each participant. Experimental results demonstrated successful cluster-based activity recognition and classification with an average regression co-efficient of 0.95 for tested clusters and features. Also, an average accuracy of $\mathbf{9 5 \%}$ was obtained for data mining models such as k-nearest neighbor, logistic regression, neural network and random forest on Evaluation Test.
\end{abstract}

Clinical Relevance-This study presents an unobtrusive (i.e., privacy-friendly) solution for activity recognition and classification, for the purposes of profiling trends in health and wellbeing.

\section{INTRODUCTION}

Recognizing individual activities of people susceptible to hazardous behaviors has been an active research topic, which has witnessed the use of pervasive and non-pervasive sensing solutions. This has also involved data gathering from laboratories, smart homes, and other home environments [1].

"Research is funded by the EU's INTERREG VA program, managed by the Special EU Program Body (SEUPB).

${ }^{1}$ School of Computing, Ulster University, BT37 0QB, Northern Ireland, United Kingdom.

${ }^{2}$ Dundalk Institute of Technology, NetwellCASALA, Dundalk Institute of Technology, Rep. of Ireland.
Interestingly, many cases of hazardous behaviors in ageing adults can be prevented [2] [3]. While there are several monitoring devices that can detect these behaviors when they occur, it would be of great benefit if they can be predicted prior to their occurrences. One way this can be achieved is with the use of machine learning models, which can help discover patterns and potential deviations from established patterns.

Pattern Deviations Assessment (PDA) in activity recognition is a vital tool in detecting abnormal activities. Its outcome helps to determine if an ageing individual can be independent or not whilst performing certain activities [4] Furthermore, activity monitoring through PDA can help determine the extent of recovery from injury, potential hazardous behavior and an individual's effectiveness. Pattern deviation can take several forms including detection of incomplete activities: sudden change in activity, disposition and posture.

Presently, Activity Recognition and Classification (ARC) in a home environment has featured many sensing solutions ranging from wearable to non-wearable. These solutions are often used to acquire data to be used in different areas, which include prediction of prevalence, and management of individuals with diseases such as dementia, osteoporosis, and increased fragility. They also help to detect hazardous incidents [5].

Nevertheless, data acquisition in a home setting can be negatively influenced by gadgets that can interfere with signal propagation from different sensing solutions. Whilst the many advantages of using a video camera for home monitoring solutions cannot be understated, lack of privacy protection, and changes in lighting conditions are the main concerns for its use.

The main contribution of this paper is to address five principal concerns in ARC: (i) wearability, (ii) privacy, (iii) actual recognition of activities, (iv) classification of activities from single to multiple users, and (v) data acquisition in a home environment. Hence, this study presents unobtrusive (i.e., privacy-friendly) sensing solutions for indoor activity

\footnotetext{
${ }^{3}$ Department of Computer Science, The University of Manchester, United Kingdom / imec-COSIC, KU Leuven, Leuven, Belgium.

${ }^{4}$ School of Computing, Electronics and Mathematics, Coventry University Coventry, United Kingdom.

${ }^{5}$ Université de Limoges, Limoges, France.

${ }^{6} \mathrm{~J}$. McLaughlin, NIBEC, Ulster University, BT37 0QB, Northern Ireland, United Kingdom
} 
recognition and classification in a home environment using data mining and fusion algorithms.

The remainder of this paper is organized as follows: Section II discusses related work; Section III examines the methodology and experiments performed; Section IV presents the data analysis and discussion; Section V presents the limitation of the study; Section VI presents conclusion and future work.

\section{RELATED WORK}

Many sensing solutions have been deployed over the years for activity recognition [5] [6] [7]. These have included the use of wearable or non-wearable solutions, or the fusion of both. In [8], it was proposed to use a hidden Markov model to recognize human activities based on data gleaned from a waist-worn accelerometer. The model also classified collected signals according to a corresponding class. Continuous monitoring was performed by a Gaussian mixture model. A further study by $\mathrm{Ni}$ et al. [9] used a multivariate online change detection algorithm for activity recognition.

The use of accelerometers for activity recognition has been featured in many studies [8] [10] [11]. In [12], the use of triaxial accelerometers was examined for monitoring ageing adults. Using binary trees, activities were classified into rest, movement, transition and emergency states. Although the successful detections of the activities were recorded, further improvements were required for successful recognition, distinction and classification of certain activities. In [13], a triaxial accelerometer was used to monitor daily physical activity. In addition to the challenges of the approach presented in [12], wearability was an issue in the latter study. Another multi-wearable sensor study was carried out by Gao et al. [10]. Whilst a garment-based accelerometer might exhibit an improved performance in a laboratory environment (as illustrated by [10]), real life usage may suffer the risks of explosion or damage to the sensors during washing activity. Also, long term usage may be a cause of discomfort for the user.

Mobile device-based ARC has also been researched in recent times. Figo et al. [14] explored the use of a smartphone's accelerometer to recognize and classify activities such as running and walking during a certain period of the day. The study incorporated information from the GPS sensor to suggest to the user routines similar to those performed in previous days. The work presented by [15] suggested that mobile devices should be optimized to enhance continuous monitoring and processing of data acquired from their sensors. Whilst these suggestions seem innovative and worthy of exploration, battery life, and users' ability to remember to carry mobile devices around are major setbacks.

Furthermore, in Konios et al. [6], a probabilistic examination of temporal and sequential aspects of activities using an approach based on the cumulative distribution function is employed to determine abnormalities in activities. This approach involved deriving probabilities of abnormal behaviors with respect to the duration and the stages of an activity. Whilst this study introduced an effective way to detect (ab)normal activities, a profile analysis of users aimed at ensuring more precision in detecting the presence of healthrelated abnormalities is still being researched.

Data fusion from homogeneous and heterogenous sensors has also been deployed in ARC. Garcia-Constantino et al. [16] investigated the fusion of data from wearable (accelerometer) and ambient (thermal) sensors by extracting relevant features from both. Initial results from this approach indicated an improvement in recognizing the main steps associated with activities.

Machine learning models have positively influenced ARC. These have included successful recognition and classification of data obtained from controlled trials in ARC. Whilst many monitoring models can exhibit excellent performance in a controlled environment such as laboratories [9], others can only be moderated by trained personnel [17]. This often results in successful laboratory work which cannot be deployed in a real-life setting. Unlike some of the existing technologies and models in ARC, this study addresses some of the concerns associated with wearability, privacy of users, actual recognition of activities in a home environment, and classification of some activities from single to multiple users.

\section{MeThodOLOGY AND EXPERIMENTS}

The present work uses two infrared thermopile array sensors to monitor and recognize activities in a laboratory kitchen, which is similar to a home kitchen. The two thermal sensors are used simultaneously to address instances of missing thermal blobs due to occlusion. Data mining tools and algorithms are used to extract features and to fuse data from both sensors. Contact sensors are used as the baseline to compare the timestamps of both types of sensors.

The study was carried out at a laboratory kitchen (Fig. 1), which measures $3.9 \mathrm{~m}$ by $3.4 \mathrm{~m}$. 10 healthy participants took part in the study and each of them participated in a single experiment. Prior to the study, the participants were briefed on the activities and their consents were obtained. Moreover, in order to have a more realistic scenario, they were allowed to take as long as they wished to complete the activities. There were no time constraints or control on the duration of the activities undertaken.

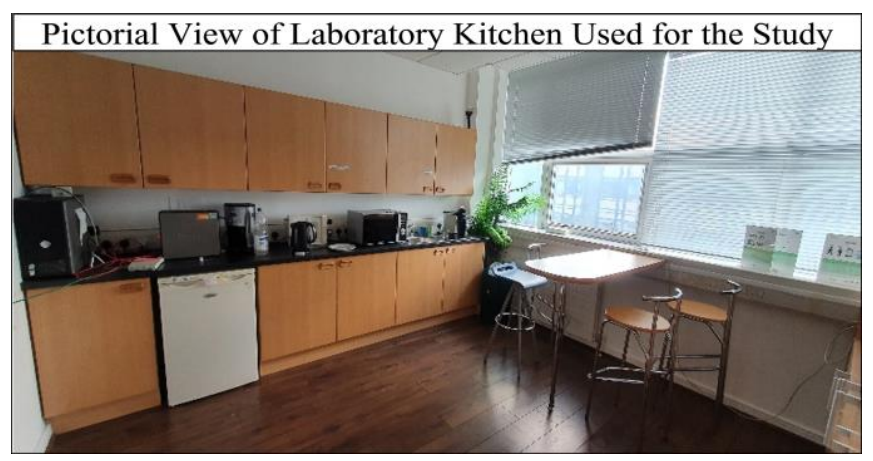

Figure 1. Pictorial View of the laboratory kitchen used for the study. Detailed description of the kitchen layout is presented in Figure 2.

The laboratory kitchen is comprised of cupboards (labelled 1 -4 in Fig. 2) where tea, coffee, cups and sugar were stored. Underneath the cupboards is a worktop with a microwave, a 
kettle and a sink over it, thus mimicking a real-life kitchen. A refrigerator is located on the floor beneath the worktop, as indicated in Fig. 2. The main kitchen area is where participants walked around to prepare a hot beverage (either tea or coffee) which was then taken to the table area for consumption.

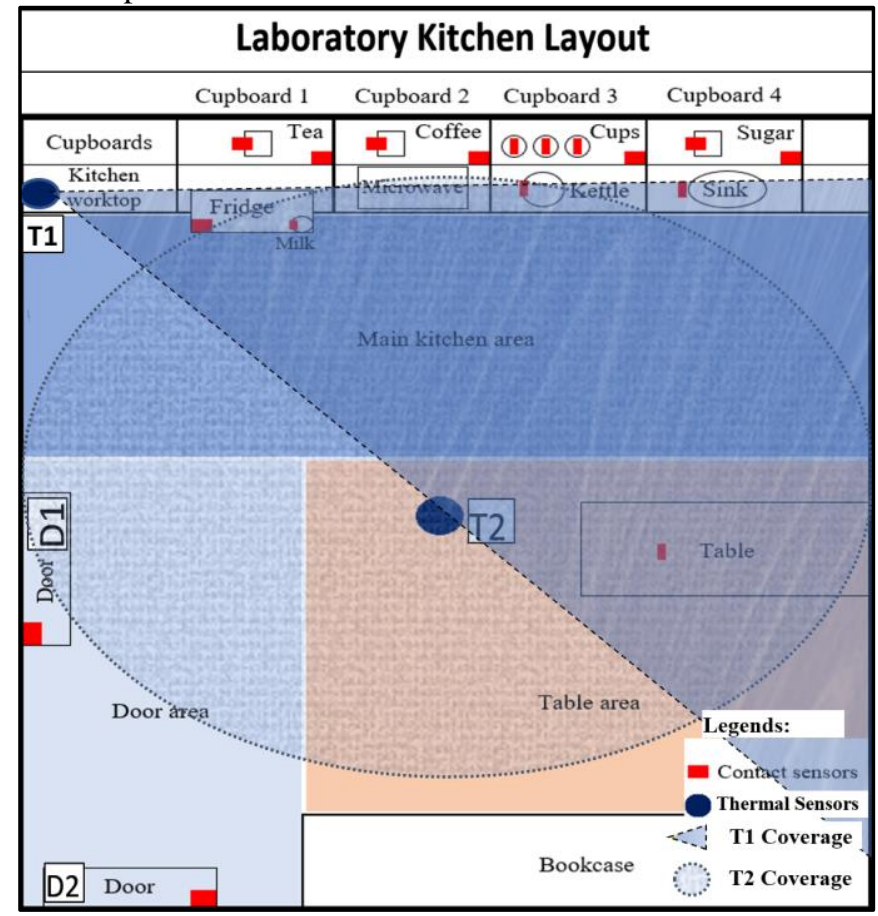

Figure 2. Laboratory Kitchen Layout. The areas marked in red indicate the location of the contact sensors. Thermal sensors are indicated by the navyblue oval shape as T1 and T2 for lateral and ceiling thermal sensors, respectively. The indicative coverages of $\mathrm{T} 1$ and $\mathrm{T} 2$ are also indicated.

During data acquisition, each participant (at a time) walked in through door D1 to the main kitchen area where the cups were located. While some participants preferred to boil water in the kettle before going for the cups, others did the opposite. Data acquisition began a few seconds prior to opening door D1 notwithstanding the activity preferences of the participants.

Moreover, data from $\mathrm{T} 1$ and $\mathrm{T} 2$ were collated using a bespoke time series database referred to as SensorCentral [18] [19] [20]. A total of 11,980 frame data (1,198 from each participant) were gleaned from the database per second from the 10 experiments. The contact sensors, which were also associated with the database, were able to record the times when each activity began and ended.

\section{DATA ANALYSIS AND DisCUSSION}

Prior to sensor data fusion, data obtained from the database were situated in two folders, each representing sensors T1 and $\mathrm{T} 2$. Thermal blobs from T1 clearly have the ability to identify and distinguish between activities such as using a bottle of milk from using a kettle of hot water (Fig. 3). While a bottle of milk was seen as monochromatic shades of black due to its low temperature, a kettle of hot water had shades of white representation due to its high temperature as presented in Fig. 3. Moreover, it is important to note that notwithstanding the closeness of the participants to the thermal sensor (Fig. 3), their identities were still protected.

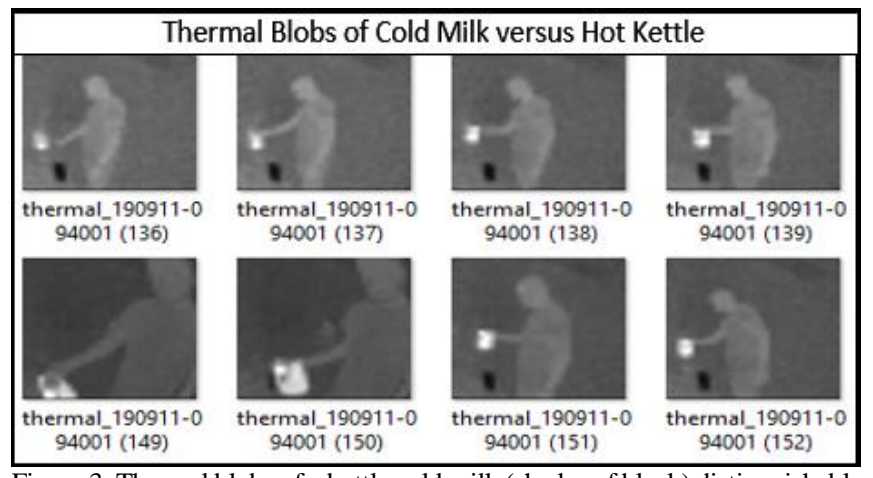

Figure 3. Thermal blobs of a bottle cold milk (shades of black) distinguishable from a hot kettle (shades of white).

Furthermore, after preparing a cup of tea, it was easier to know from the thermal blobs whether the user successfully reached the table. Also, it was necessary to know where the participant placed the hot kettle (after using it) which is a potential hazardous object. As presented in Fig. 4, these activities were clearly viewed on the thermal image. Whilst the hot kettle was represented as a large blob adjacent to the participant, the tea/coffee cup was viewed as a small bright spot in what could be viewed as the hand of the user (Fig. 4). Thermal Blobs Distinguishing a Hot Kettle from a Cup of Tea/Coffee

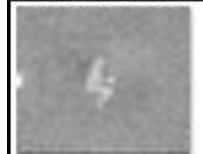

thermal_190

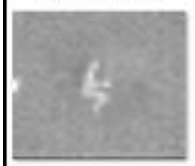

thermal_197
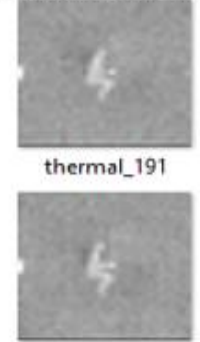

thermal_198
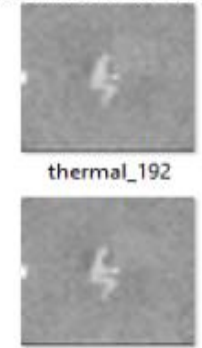

thermal_199

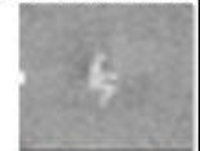

thermal_193

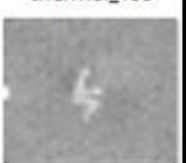

thermal_200
Figure 4. Distinguishable thermal blobs of hot kettle, position of participant and tea/coffee cup after the initial act of tea/coffee making.

At some instances, the heat spot of a cup or kettle may be occluded by a participant when it is viewed from the lateral thermal sensor. When this happens, an abnormal behavior or an activity may go unnoticed. To address these concerns, the ceiling sensor (T2) is referred to for an aerial view as presented in Fig. 5. Hence, the essence and usefulness of dual sensing in this study.

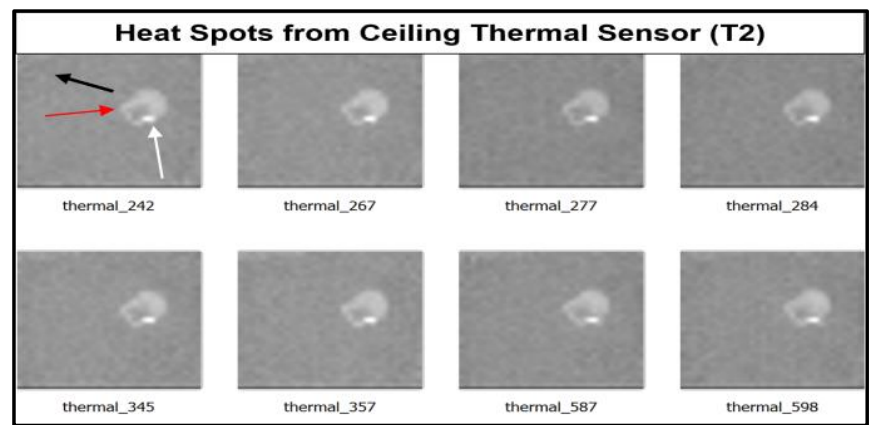

Figure 5. Heat Spots from tea/coffee cups occluded from the lateral thermal sensor (T1) but indicated by the ceiling thermal sensor (T2). The black arrow on thermal_242 points to the location of T1; the white arrow points to the heat spot and the red arrow points to the hand of the participant (occluding the heat spot). 


\section{A. Fusion of Thermal Blobs from Participants}

Sensor fusion using data mining tools helps to merge data, and to extract and cluster features from both sensors. A block diagram of the sensor data fusion algorithm employed in this study is presented in Fig. 6 [21].

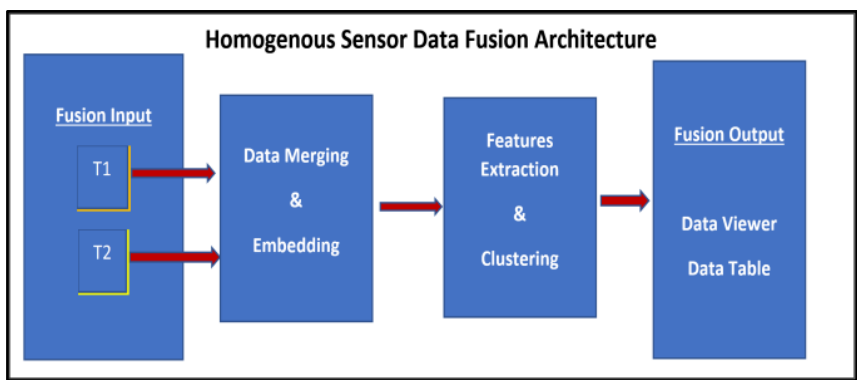

Figure 6. Sensor Data Fusion Architecture for lateral (T1) and ceiling (T2) thermal sensors.

During sensor fusion, data from $\mathrm{T} 1$ and $\mathrm{T} 2$ were fed into the data merging system. The system then created an imaginary table for the two sets of data before carrying out a matching row appendation. This was then followed by data embedding using the SqueezeNet architecture [21], a deep neural network model for image recognition.

Unlike many sensor fusion or classification architectures that manually allocate clusters to images, the Louvain clustering algorithm [21] was used alongside distance metrics to automatically detect clusters. One of the advantages of using Louvain clustering is that of determining the number of clusters detected. The clusters were largely affected by resolution and Principal Component Analysis (PCA) parameters. In essence, increasing any of these parameters resulted in a corresponding decrease in the number of clusters that the algorithm detected. To be able to select and manipulate clusters, a separate Hierarchical Clustering Algorithm (HCA) was used. Data fusion outputs were viewed using a scatterplot, data table and data viewer widget.

Using this sensor data fusion architecture, a cluster comprising of all similar activities can be viewed as presented in Fig. 7 even if the activity was performed at different times by different participants. In Fig. 7, for example, it could be easily deduced that a participant code-named c_ID was at the kitchen table with a hot cup of tea/coffee on the 8th of May, 2019 at a different time and date as another participant codenamed c_OR. With this information, activities can be easily monitored in clusters notwithstanding the times and dates they were performed.

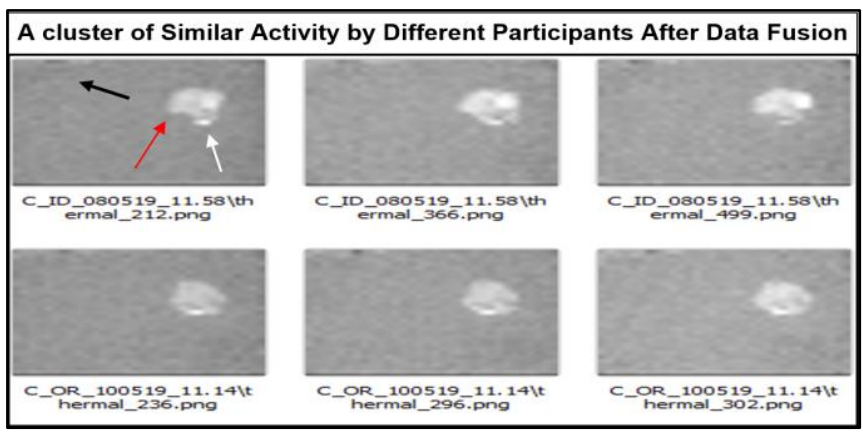

Figure 7. A cluster of data fusion output showing thermal blobs from two participants in a cluster with timestamps. The black arrow on 'C_ID_080519_11.58 \thermal_212.png' points to the location of the lateral sensor; the red arrow points to the participant and the white arrow points to the heat spot from tea/coffee cup.
Furthermore, it is important to note that up to 1,000 features (labelled n0 to n999) were extracted from each thermal image during the feature extraction process. Using these features, a PCA and scoring of the clusters performed between features $\mathrm{n} 525$ and $\mathrm{n} 830$ at $99 \%$ variance coverage indicated a regression co-efficient (r) of 0.98 and 1.00 for clusters 2 and 12, respectively as presented in Fig. 8.

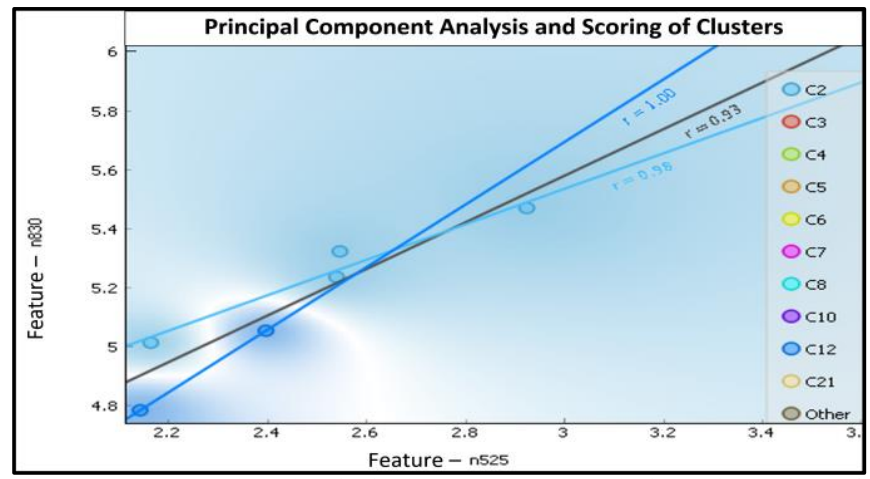

Figure 8. Features-based Principal Component Analysis and Scoring of Clusters. Features $\mathrm{n} 525$ and $\mathrm{n} 830$ are indicated on the $\mathrm{X}$ and $\mathrm{Y}$ axes, respectively. The clusters are color-coded, and the color of each regression line on the graph matches the color on the cluster legend on the right.

Similarly, a PCA and scoring analysis performed between features $\mathrm{n} 246$ and $\mathrm{n} 170$ for clusters 1,6 and 9 yielded 0.83 , 0.99 and 1.00 as presented in Fig. 9. These resulted in an average (r) of 0.95 for all the tested features and clusters which were randomly selected from the HCA interface.

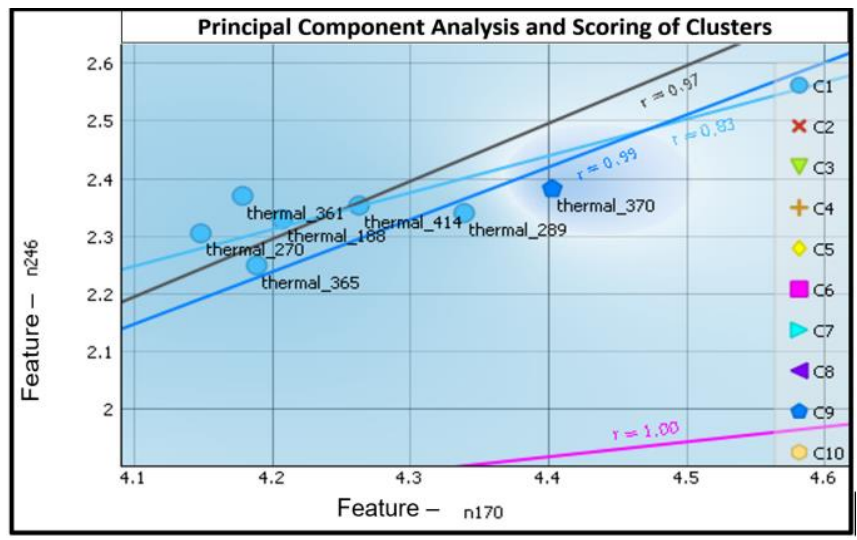

Figure 9. Features-based Principal Component Analysis and Scoring of Clusters. Features $\mathrm{n} 170$ and $\mathrm{n} 246$ are indicated on the X and Y axes, respectively. Also, the clusters are color-coded and the color of each regression line on the graph matches the color on the cluster legend on the right.

To further ascertain the certainty of the predicted clusters, an Evaluation Test was performed on all the clusters in the HCA using K-Near Neighbors (KNN), Logistic Regression (LR), Neural Network (NN) and Random Forest (RF) models. While KNN yielded the least Classification Accuracy (CA) of 0.85 , LR and NN gave CAs of 0.961 and 1.00 respectively as presented in Table 1. Also, the proportion of true positives of the positively classified instances (Precision) [21] followed a similar trend as the CA. In addition, the NN yielded a value of 1.00 for Area Under the Curve (AUC), weighted average (F1), CA, Precision, Recall and Specificity followed by RF with an average of 0.99 as presented in Table 1 . 


\begin{tabular}{|l|llllllll|}
\hline \multicolumn{7}{|c|}{ TABLE 1 } \\
\hline \multicolumn{1}{|c|}{ Eodel } & AUC & CA & F1 & Precision & Recall & Logloss & Specificity \\
\hline kNN & 0.991 & 0.850 & 0.850 & 0.854 & 0.850 & 0.355 & 0.983 \\
Logistic Regression & 0.999 & 0.961 & 0.961 & 0.961 & 0.961 & 0.186 & 0.996 \\
Neural Network & 1.000 & 1.000 & 1.000 & 1.000 & 1.000 & 0.001 & 1.000 \\
Random Forest & 1.000 & 0.989 & 0.989 & 0.989 & 0.989 & 0.282 & 0.999 \\
\hline
\end{tabular}

LogLoss, also referred to as Cross entropy loss, accounts for the performance of the classification model with respect to its variation from the actual label [21], and was relatively low (less than 0.4 ) for all the models (Table 1). NN had the least value of 0.001 .

With an average regression co-efficient of 0.95 in the PCA and scoring test and $95 \%$ accuracy for CA, AUC, F1, Precision, Recall and Specificity in the Evaluation Test, the predictive accuracy of this novel fusion architecture is well demonstrated.

\section{LIMITATION OF THE STUDY}

One of the limitations of this study is that the thermal sensors leveraged on the contact sensors to determine if an occupant drank tea or coffee during the experiments since both (tea and coffee) were placed in the same locker. This implies that depending on the data from the thermal sensors alone, it would be difficult to determine if an occupant had tea or coffee. In a real-life setting, however, this confusion can be resolved if tea and coffee are placed on separate lockers that are more than $1 \mathrm{~m}$ apart.

Another challenge with using the thermal sensors only without the contact sensors is on determining if the occupant used milk or cold water if both are placed in a similar container. To address this limitation in a real-life application, milk and cold water should be placed in containers of different sizes so that their blobs could be easily differentiated.

\section{CONCLUSION AND FUTURE WORK}

This paper presented the use of low-cost unobtrusive (privacy-friendly) sensing solutions for indoor ARC in a laboratory kitchen environment similar to a home environment. Experimental results indicated instances of activity recognition during activities such as making a cup of tea/coffee and classification of the same activities using data mining tools with an average predictive accuracy of $95 \%$. Future work will calculate the speed and range of these activities including the use of data mining tools to score and evaluate their performance.

\section{REFERENCES}

T. T. Truyen, H. H. Bui, D. Q. Phung, and S. Venkatesh, "Learning Discriminative Sequence Models from Partially Labelled Data for Activity Recognition BT - PRICAI 2008: Trends in Artificial Intelligence," 2008, pp. 903-912.

[2] M. K. Karlsson, H. Magnusson, T. Von Schewelov, and B. E. Rosengren, "Prevention of falls in the elderly-a review," Osteoporos. Int., vol. 24, no. 3, pp. 747-762, 2013, doi: 10.1007/s00198-012-2256-7.

[3] A. H. Myers, Y. Young, and J. A. Langlois, "Prevention of falls in the elderly," in Bone, 1996, doi: 10.1016/8756-3282(95)00384-3.
C. Debes, A. Merentitis, S. Sukhanov, M. Niessen, N. Frangiadakis, and A. Bauer, "Monitoring activities of daily living in smart homes: Understanding human behavior," IEEE Signal Process. Mag., vol. 33, no. 2, pp. 81-94, 2016, doi: 10.1109/MSP.2015.2503881.

[5] M. Garcia-Constantino, A. Konios, and C. Nugent, "Modelling Activities of Daily Living with Petri nets," 2018 IEEE Int. Conf. Pervasive Comput. Commun. Work. PerCom Work. 2018, no. i, pp. 866-871, 2018, doi: 10.1109/PERCOMW.2018.8480225.

[6] M. Garcia-Constantino, A. Konios, I. Ekerete, S. R. G. Christopoulos, C. Shewell, C. Nugent, and G. Morrison, "Probabilistic Analysis of Abnormal Behaviour Detection in Activities of Daily Living,” 2019 IEEE International Conference on Pervasive Computing and Communications Workshops,

PerCom Workshops 2019, pp. 461-466, 2019.

[7] I. Ekerete, C. Nugent, and J. Mclaughlin, "An unobtrusive sensing solution for home based post-stroke rehabilitation.," 2018 IEEE Int. Conf. Bioinforma. Biomed., pp. 1478-1479, 2018.

[8] J. Wang, R. Chen, X. Sun, M. F. H. She, and Y. Wu, "Recognizing human daily activities from accelerometer signal," Procedia Eng., vol. 15, pp. 1780-1786, 2011, doi: 10.1016/j.proeng.2011.08.331.

[9] Q. Ni, T. Patterson, I. Cleland, and C. Nugent, "Dynamic detection of window starting positions and its implementation within an activity recognition framework," J. Biomed. Inform., vol. 62, pp. 171-180, 2016, doi: 10.1016/j.jbi.2016.07.005.

[10] L. Gao, A. K. Bourke, and J. Nelson, "Evaluation of accelerometer based multi-sensor versus single-sensor activity recognition systems," Med. Eng. Phys., vol. 36, no. 6, pp. 779-785, 2014, doi: 10.1016/j.medengphy.2014.02.012.

[11] B. Fida, I. Bernabucci, D. Bibbo, S. Conforto, and M. Schmid, "Varying behavior of different window sizes on the classification of static and dynamic physical activities from a single accelerometer," Med. Eng. Phys., vol. 37, no. 7, pp. 705-711, 2015, doi: 10.1016/j.medengphy.2015.04.005.

[12] D. W. Kang, J. S. Choi, J. W. Lee, S. C. Chung, S. J. Park, and G. R. Tack, "Real-time elderly activity monitoring system based on a tri-axial accelerometer," Disabil. Rehabil. Assist. Technol., vol. 5, no. 4, pp. 247-253, 2010, doi: 10.3109/17483101003718112.

[13] C. V. C. Bouten, K. T. M. Koekkoek, M. Verduin, R. Kodde, and J. D. Janssen, "A triaxial accelerometer and portable data processing unit for the assessment of daily physical activity," IEEE Trans. Biomed. Eng., vol. 44, no. 3, pp. 136-147, 1997, doi: $10.1109 / 10.554760$.

[14] D. Figo, P. C. Diniz, D. R. Ferreira, and J. M. P. Cardoso, "Preprocessing techniques for context recognition from accelerometer data," Pers. Ubiquitous Comput., vol. 14, no. 7, pp. 645-662, 2010, doi: 10.1007/s00779-010-0293-9.

[15] J. W. Lockhart, T. Pulickal, and G. M. Weiss, "Applications of mobile activity recognition," UbiComp'12 - Proc. 2012 ACM Conf. Ubiquitous Comput., pp. 1054-1058, 2012, doi: 10.1145/2370216.2370441.

[16] M. Garcia-Constantino, A. Konios, C. Nugent, and G. Morrison, "Ambient and Wearable Sensor Fusion for Abnormal Behaviour Detection in Activities of Daily Living," In 5th IEEE PerCom Workshop on Pervasive Health Technologies, 2020.

[17] T. Shany, S. J. Redmond, M. Marschollek, and N. H. Lovell, "Assessing fall risk using wearable sensors: a practical discussion," Z. Gerontol. Geriatr., vol. 45, no. 8, pp. 694-706, 2012, doi: 10.1007/s00391-012-0407-2.

[18] I. Cleland, S. McClean, J. Rafferty, J. Synnott, C. Nugent, A. Ennis, P. Catherwood, I. McChesney, "A Scalable, Research Oriented, Generic, Sensor Data Platform," IEEE Access, vol. 6, pp. 4547345484, 2018.

[19] I. Ekerete, C. Nugent, O. Giggins, and J. McLaughlin, "Unobtrusive Sensing Solution for Post-stroke Rehabilitation," in Smart Assisted Living. Computer Communications and Networks, N. C. Chen F., García-Betances R., Chen L., Cabrera-Umpiérrez M., Ed. Springer, Cham, 2020, pp. 43-62.

[20] I. Ekerete, C. Nugent, and J. Mclaughlin, "An unobtrusive sensing solution for home based post-stroke rehabilitation."

[21] P. Bhatia, "Introduction to Data Mining," Data Min. Data Warehous., no. May, pp. 17-27, 2019, doi: 10.1017/9781108635592.003. 